\title{
AN APPROACH TO RESILIENCE IN TRANSPORTATION PLANNING
}

\author{
I.M. SCHOEMAN \\ Unit for Business Mathematics and Informatics, North West University (Potchefstroom Campus), South Africa.
}

\begin{abstract}
Dynamic systems are characterize by a collection of variables and their interrelationships over time. Hence, a dynamic system refers to anything that evolves or changes over time like your bank account or a countries economic growth. Resilience is the ability of a dynamic system to return to a steady state or stable periodic orbit after a (not to big) disturbance and therefore the systems behaviour will be stable or marginally stable. Resilience behaviour of the system strives to minimize regret and mitigate risk by being a stable or marginally stable system. In mathematical terms this definition of resilience refer to convergence, for all starting values near the equilibrium (or small disturbance to the equilibrium) the system will not move away from the/a equilibrium, i.e. a stable (steady state) system or the stable periodic orbit. Hence the system will oscillate between a finite number of points on the long term.

To achieve sustainability within a system (e.g. transportation networks, etc.), the way of thinking, planning, project design and implementation needs to be resilient in order to contribute to the system wide sustainability. It thus implies a need for quantitative data and information to optimise planning and to support decision making in an adaptive fashion. Through being able to describe how the system evolves over time, it enable ways to define preconditions or input that will ensure a dynamic system remain stable to promote resilience and sustainability. At present, theory and practices do not make provision for the development of improved adaptive capacities in all phases of planning through dynamic transportation systems planning and development.

The aim of this paper is to introduce and develop resilience-orientated frameworks and approaches based on application of mathematical, statistical and decision-making tools, which can be used to enhance the interface between resilience and sustainability alignment though dynamic thinking, planning and implementation in transportation systems. In the end, this will lead to improved management and sustainable transport planning.

Keywords: adaptive capacity and decision making, dynamic planning, dynamic transportation systems, resilience, sustainability.
\end{abstract}

\section{INTRODUCTION}

This paper assumes that the system and its parameters are known and aim to achieve optimal decision making and uncertainty reduction through resilience. It should be noted that resilience is not a once of happening but must be actively manage in the dynamic reality. Derissen et al. [1] refer to sustainability as a way to mitigate change by keeping resources above static safe levels, and on the other hand with resilience the safety levels is more dynamic but will also ensure that the system will return to a steady state after the disruption.

From the work of Schoeman [2] and Schjetlein [3] follow, that one of the main goals of development (or social change) must be or is sustainability and that it is the result of a resilience way of acting and thinking. Therefore, to achieve resilience and sustainability in decisions, choices, etc. planning and implementation must be undertaken using logic, analytics and probabilities. In addition, according to Berdica [4] to implement sustainable, applicable and resilient policies in transport studies, there is a need for insight into:

- resulting consequences;

- vulnerabilities or propensity to malfunction;

- mitigation measures. 


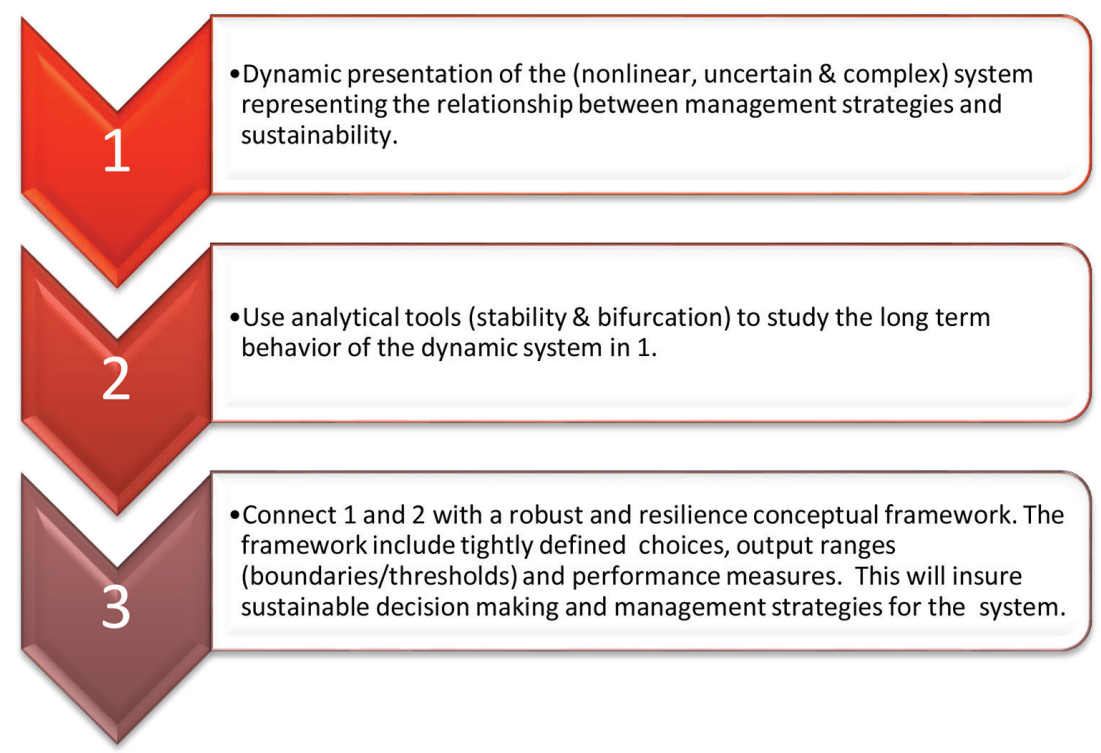

Figure 1: The alignment of robustness, resilience and sustainability. Source: Own construction from Anderies et al. [5].

Anderies et al. [5] align robustness, resilience and sustainability to address multi-level and multi-scale challenges associated with global change. For a summary of this alignment, consider Fig. 1, where resilience refer to the enhancement of the understanding how the complex system change and self-organize over time. Therefore, resilience enriches the system management strategy and supports the sustainable decision-making goal.

The paper will illustrate the connection between dynamic system and its long-term behaviour. Therefore, resilience can be conceptualized and specifically defined.

\section{BEHAVIOUR OF A DYNAMIC SYSTEM}

In the dynamic system, the current output depends on current and/or past inputs/outputs/error terms and the output takes time to react. The aim of this section is to provide the reader with an overview and insight into supporting analytical tools to study the long-term behavior of the dynamic system and decision making. Different types of models can be used to describe dynamical systems.

\subsection{The deterministic dynamic system}

From Scheinerman in Ref. [6] follows that in the deterministic dynamical system the state vector denotes the current state of the system (summarizes all past information that is relevant for the future), and the rule (function) tells us given the current state, what will be the longterm behaviour of the system. In Fig. 2, the function, $f$, is virtually any nonlinear, differentiable function with continuous derivatives. The function, $f$, gives information on the changes/ movement (if any) within the (one or multidimensional) dynamic system, therefore in the deterministic dynamical system the future state of the system is fixed. The state vector contains a finite number of elements in a finite-dimensional system. 


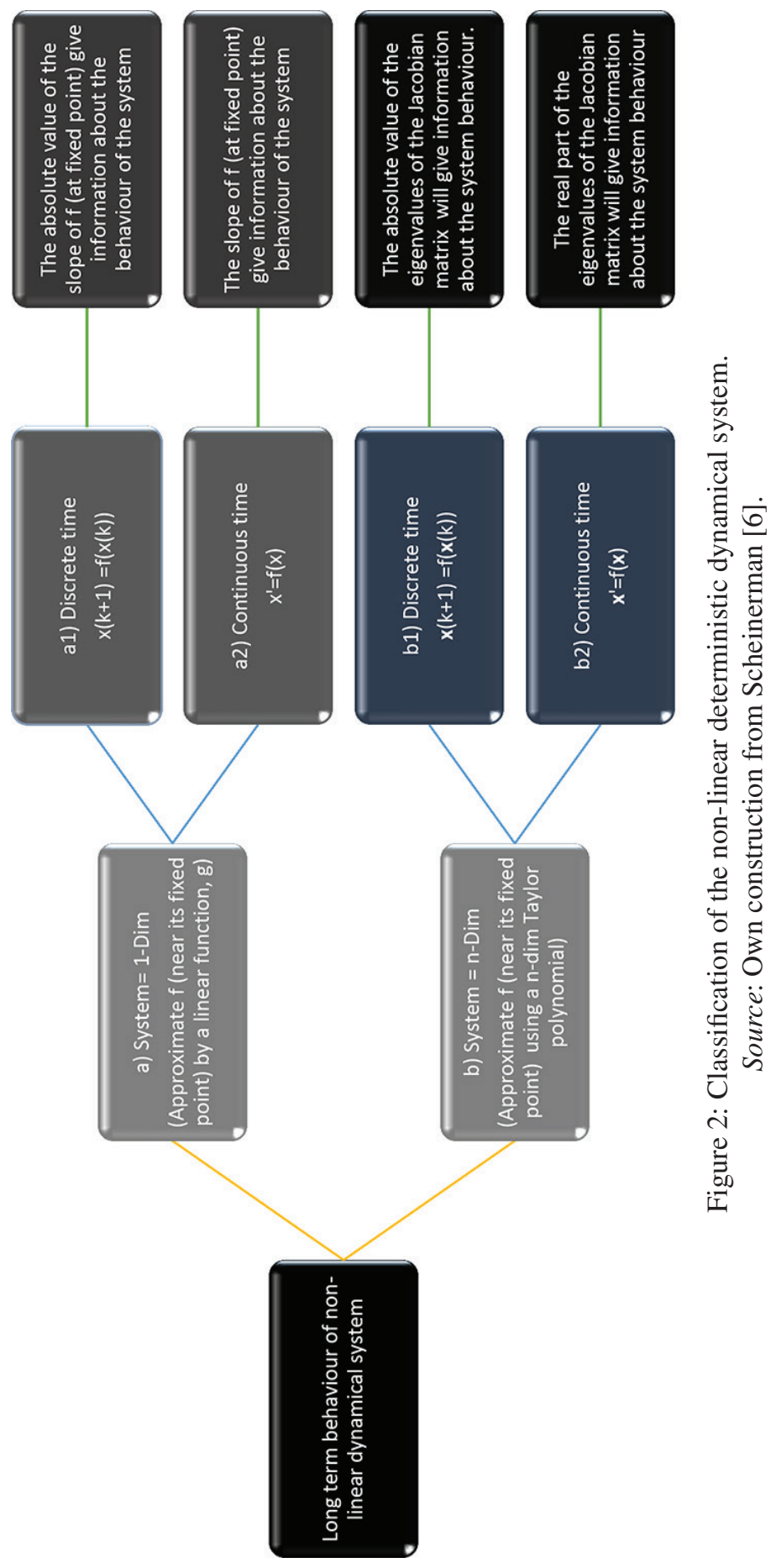


2.1.1 The long-term behavior of the one-dimensional dynamic system

The long-term behavior of the one-dimensional deterministic dynamic system will be stable, marginal stable (oscillates) or unstable.

a) Consider the discrete linear one-dimensional system $x_{t+1}=a x_{t}+b, x_{0}=$ Given. For resilience the long-term behavior will be stable if

$$
|a|<1 \text { or }\left(|a|>1 \text { and } x_{0}=\frac{b}{1-a}\right) \operatorname{or}(a=1 \text { and } b=0) \text { or }\left(a=-1 \text { and } x_{0}=\frac{b}{2}\right) \text {. }
$$

and marginally stable (oscillation) if $x_{0} \neq \frac{b}{2}$ and $a=-1$. Therefore, unstable behavior will follow if $\left(|a|>1\right.$ and $\left.x_{0} \neq \frac{b}{1-a}\right) \operatorname{or}(a=1$ and $b \neq 0)$.

b) Consider the continuous linear one-dimensional system $\mathrm{x}^{\prime}=\mathrm{a} x_{t}+\mathrm{b}, x_{0}=$ Given. For resilience long-term behavior in the continuous time system the following must be true

$$
a<0 \text { or }\left(a>0 \text { and } x_{0}=\frac{-b}{a}\right) \text { or }(a=0 \text { and } b=0) .
$$

Unstable behavior will be the result if ( $a>0$ and $x_{0} \neq \frac{-b}{a}$ )or $(a=0$ and $b \neq 0)$.

c) The long-term behavior of the discrete non-linear one-dimensional system:

A fixed point, $\dot{x}$ of a dynamical system is the equilibrium point of the dynamical system. In the neighborhood of a fixed point approximate the non-linear function, $f$ by a linear function, say $g=f^{\prime}(x)(x-\dot{x})+f(\dot{x})$. The discrete non-linear one-dimensional system with stable (resilience) long-term behavior will be the one for with $\left|\mathrm{f}^{\prime}(\dot{\mathrm{x}})\right|<1$ and for unstable long-term behavior $\left|\mathrm{f}^{\prime}(\dot{\mathrm{x}})\right|>1$.

d) Consider the continuous non-linear one-dimensional system $x$ ' $=f(x), f=$ non - linear. For (resilience) long-term behavior in the continuous time system if $\mathrm{f}^{\prime}(\dot{\mathrm{x}})<0$ and unstable behavior will be the result if $f^{\prime}(\dot{x})>0$.

2.1.2 The long-term behavior of the multi-dimensional deterministic dynamic system Approximate the non-linear function, $\mathrm{f}$ by a linear function, say $\mathrm{H}(\dot{\mathbf{x}})(\mathbf{x}-\dot{\mathbf{x}})+\mathrm{f}(\dot{\mathbf{x}})$ in the neighborhood of a fixed point, $\dot{\mathbf{x}}$. Here $\mathrm{H}(\dot{\mathbf{x}})$ is the Jacobian matrix of f, i.e. the matrix of partial derivatives of $\mathrm{f}$. In the $n$-dimensional system case, the long-term behavior will be:

- stable or

- stable periodic orbit if it is in one of the following states

- marginal stable (circular orbit),

- the same states infinitely often (inwards spiral or outward spiral approaching a stable cycle),

- the system can be decomposed into periodic subsystems;

- unstable, i.e. the system explodes to infinity.

- unstable periodic orbit (spiral outward to infinity) use bifurcation in the discrete time dynamical system to find these points by altering the function over time.

- chaotic (non-periodic and nonexplosive) behavior, i.e. slight modification in the initial value yields enormous fluctuations in subsequent iterations (sensitive dependence on initial conditions). 
a) Consider the discrete linear n-dimensional system $\boldsymbol{x}_{t+1}=\mathrm{A} \boldsymbol{x}_{t}+\mathbf{b}, \boldsymbol{x}_{0}=$ Given. Stable (resilience) long-term behavior in the multi-dimensional discrete time system if all the eigenvalues of matrix $A$ are in the open unit disc (i.e. $\left|\lambda_{i}\right|<1$, for all i) and marginal stable behavior if all the eigenvalues of the matrix A are in the closed unit disc. The system will be unstable if there is an eigenvalue outside the closed unit disk.

b) Consider the continuous linear n-dimensional system $\mathbf{x}^{\prime}=\mathrm{A} \boldsymbol{x}_{t}+\mathbf{b}, \boldsymbol{x}_{0}=$ Given. For (resilience) long-term behavior in the multi-dimensional continuous time, system if A has all its eigenvalues in the open left half plane (i.e. real part of $\lambda_{i}<0$ ) and unstable behavior otherwise.

c) Study the discrete non-linear n-dimensional system $\boldsymbol{x}_{t+1}=\mathrm{f}\left(\boldsymbol{x}_{t}\right)$. Resilience (stable) long-term behavior in the multi-dimensional discrete time system will depend on the classification of the fixed points. For a fix point $\dot{\mathbf{x}}$ if all the eigenvalues of Jacobian matrix $\mathrm{H}(\dot{\mathbf{x}})$ are in the open unit disc (i.e. $\left|\lambda_{i}\right|<1$, for all $\left.i\right)$ then the fix point will be stable. The fixed point will be unstable if there is an eigenvalue outside the closed unit disk. The test fails if there is an eigenvalue on the closed unit disk, in this case a Lyapunov function can be used for the classification of the fixed point.

d) In the continuous non-linear n-dimensional system $x^{\prime}=f(x), f=$ non-linear. If the eigenvalues of the Jacobian, $\mathrm{H}(\dot{\mathrm{x}})$ all have negative real part, the fixed point is stable and if their exists some eigenvalues that has positive real part then the fixed point is unstable. Consider Fig. 2 for a summary of the classification of non-linear systems. Note that in engineering terms a conservative vector field (gradient vector) will be stable (resilience) in a point if its rotation is zero in that point.

\subsection{The stochastic dynamic system}

A stochastic process is a model for a random variable that is observed over time, i.e. a collection of random variables one for each time $t$ in some index set, consider Hull [7]. Therefore, a stochastic process is a function of both time and randomness and the future outputs can only be predicted with some error. If the change/growth does not progress in some rigorously predefined deterministic fashion (i.e. the next state is random), then the dynamic system is stochastic. Figure 3 gives the classification of stochastic processes.

In transport, one uses mostly discrete time models, since the available data are often observed in discrete time. Hence, the stochastic dynamical Markov chain and the time-series models play an import role in transport. Time-series data consisting of observations of one or more variables over time that is arrange in chronological order. Time-series models

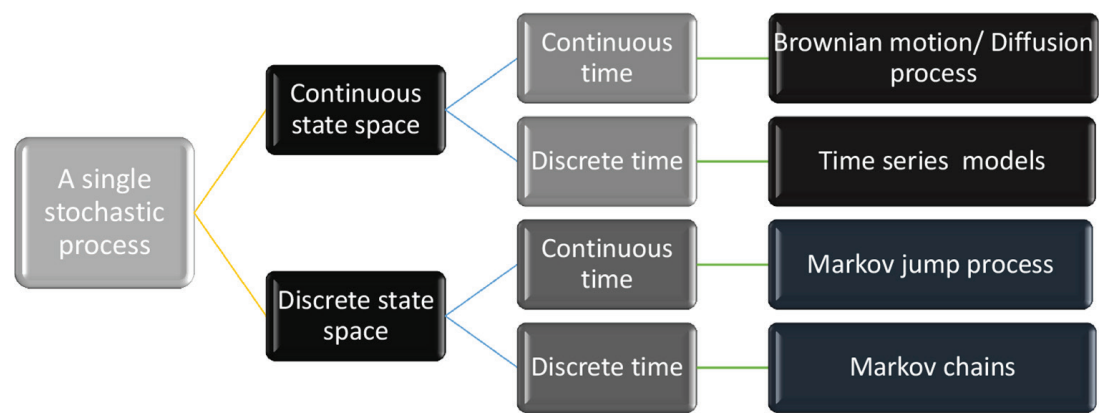

Figure 3: Taxonomy of stochastic dynamic systems.

Source: Own construction from [7]. 
contain the following models. Distributed lag models, which include lagged terms of the explanatory variable, $Y_{t}=\alpha+\beta_{0} X_{t}+\beta_{1} X_{t-1}+\beta_{2} X_{t-2}+\ldots+\beta_{p} X_{t-p}+u_{t}$. Autoregressive, $\mathrm{AR}(\mathrm{p})$ models include lagged terms of the dependent variable, i.e. $Y_{t}=\varnothing_{1} Y_{t-1}+\varnothing_{2} Y_{t-2}+\ldots+\varnothing_{p} Y_{t-p}+u_{t}$. The Moving Average, MA(q) model is of the form: $Y_{t}=u_{t}+\theta_{1} u_{t-1}+\theta_{2} u_{t-2}+\ldots+\theta_{q} u_{t-q}$ and the Autoregressive Moving Average, $\operatorname{ARMA}(\mathrm{p}, \mathrm{q})$ model form: $Y_{t}=\varnothing_{1} Y_{t-1}+\varnothing_{2} Y_{t-2}+\ldots+\varnothing_{p} Y_{t-p}+u_{t}+\theta_{1} u_{t-1}+\theta_{2} u_{t-2}+\ldots+\theta_{q} u_{t-q}$.

\subsubsection{Resilience long-term behavior in stochastic models}

The Markov chain is a stochastic system where the probability of arriving in a particular state at a particular time depends on the state at the previous time.

Consider the Markov chain $\mathbf{p}(\mathrm{m}+1)=\mathbf{p}(\mathrm{m}) \mathrm{P}$, where $\mathbf{p}(\mathrm{m})$ denotes the probability vector after $\mathrm{m}$ steps of the system and $\mathrm{P}$ is the stochastic probability matrix. The long-term behaviour of a Markov chain will be stable (resilience) if 1 is the unique eigenvalue of matrix $P$ with the largest absolute value (i.e. $\left|\lambda_{i}\right|<1$, for all $i \neq r$ and $\lambda_{r}=1$ ). The equilibrium (long-term steady state) is given by the normalised eigenvector of the transposed matrix $\mathrm{P}^{\mathrm{t}}$, which correspond to the eigenvalue $\lambda_{i}=1$. Markov chains can be used to solve queueing problems, as in Ref. [8].

A process is weakly stationary or covariance stationary if the process mean, variance and covariance remain the same over time (i.e. the correlogram that diminishes as the lag length increases). For the purpose of this paper, when we are referring to stationary, we mean weakly stationary. Note that stochastic time process/models are stationary (like White noise, Moving average, Distributed Lag Models) or non-stationary (e.g., random walk). Hence, for resilience behaviour in the time series it must be (weakly/covariance) stationary.

Long-run equilibrium between stationary stochastic variables: If all the variables are stationary and endogenous, use a Vector Auto-Regression (VAR) models for a description of the long-run equilibrium. The variance decomposition determines the significance of the impact of one variable on another. Use the impulse response function that examines the response of the dependent variable in the VAR to shocks in the error terms, i.e. whether the effect of the shock persist or dies out quickly.

The linear autoregressive distributed lag (ARDL) model determines the short-run and long-run reactions in the dependent variable after a change in the independent variable. This model also determines how quickly the equilibrium will be restored.

Long-run equilibrium between cointegrated unstationary stochastic variables: Two non-stationary variables $\left(Y_{t}\right.$ and $\left.X_{t}\right)$ are cointegrated if there exists a linear combination of these variables that is stationary. Thus the linear combination of $Y_{t}$ and $X_{t}$ is a stationary variable. Cointegration results between two or more variables confirm a long-run (steady state) equilibrium relationship between the variables and allow short-run adjustment dynamics. Hence, a long-run relationship (equilibrium) between unstationary variables $\left(Y_{t}\right.$ and $\left.X_{t}\right)$ indicates the existence of interdependence between variables. A steady state between different variables play an important role:

- in the implementation and planning of policies;

- in identifying the variables related to a crisis;

- forecasting.

The Error-Correction Mechanism/Model (ECM) is a means of reconciling the short-run behaviour of two variables with its long-run behaviour if the variables are cointegrated. The ECM specifications measure the correction from disequilibrium of the previous period. 
In the case where more than two variables are cointegrated use the Vector Error-Correction Model (VECM) in the estimation of the short and long-run equilibrium parameters. To forecast and analyse the dynamic effect of the variables in the VECM use the Variance Decomposition and Generalized Impulse Response Function (GIRF).

Note that before proceeding with the dynamic analysis of an estimated Statistical or Dynamical Model perform diagnostic tests and checks to determine if the model fits all the model assumptions. Also, ensure that the time-series data is without structural breaks. The importance of stationarity is that if a process is non-stationary, all the typical results of the classical regression analysis and time-series models like VAR, Autoregressive (AR) and Autoregressive Moving Average (ARMA) model are not valid.

For further and more information on this section, refer to Anderson et al. [9], Asteriou and Hall [10], Gujarati and Porter [11], Chu [12], Oriavwote and Eriemo [13].

\section{OPTIMAL DECISION MAKING}

This section considers modelling and analysis methods by which uncertainty can be reduced or predicted. This can be done by using Monte Carlo simulation to evaluate the impact of uncertainty and for scenario planning to enhance decision making and management.

\subsection{Modelling and analysis}

Applicable intervention and inform management are the result of analysis and modelling. Analysis, assessment, simulation and modelling help the managers or decision makers to understand what happened in the past, identify trends over time and predict future values based on past patterns.

Monte Carlo simulation contribute to better decision making when uncertain quantities complicate the decision process since it estimated the likelihood of (desirable) outcomes for the uncertain variables that can be used in scenario planning and to access the risk of an unwanted outcome. Hence, Monte Carlo provides the decision maker with the probability of all outcomes/results (for the uncertain input) it also help the decision maker in determining, which inputs had the main effect on the results. Schoeman [14] use the Monte Carlo method to predict 2016 traffic accidents in South Africa. It follows from Fig. 4 that the probability of

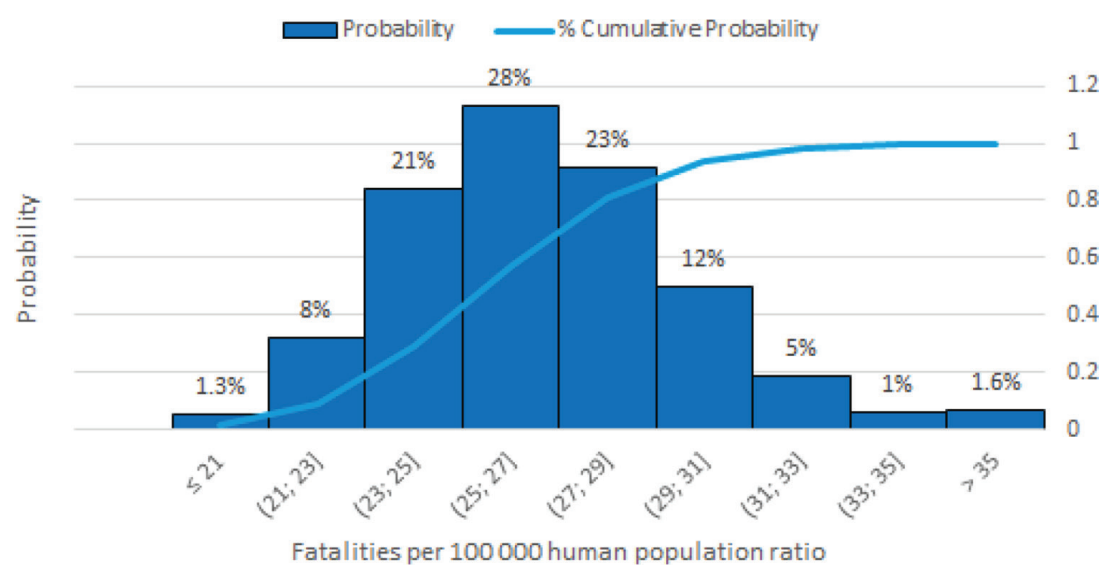

Figure 4: The predicted traffic accident fatalities per 100000 human population. Source: Ref. [14]. 
having a fatalities per 100000 human population ratio in the interval $(25 ; 27]$ is the highest at $28 \%$ and the profitability of having this ratio higher than 25 is equal to $71 \%$. Thus, the Monte Carlo simulation imply that the probability is very high in 2016 for the realisation of a mortality rate greater than 24 .

Hull [7] calculate the market risk using the Value-at-risk (VaR) at a $99 \%$ confidence level over the (back testing) period. Therefore, if a portfolio has a $99 \%$ weekly value of risk of R1.25 million it would imply the worst-case scenario risk is R1.25 million since there is a $99 \%$ chance that the portfolio will lose no more than R1.25 million.

The ARCH and GARCH time-series models in Ref. [11] model the attitude of investors towards expected return and risk (uncertainty). These models assume the current volatility is at most a function of past data.

\subsection{Optimal control}

Note that control methods were commonly developed for linear systems and in many cases can nonlinear systems be approximated by linear systems. The two main approaches to study control theory are given in Fig. 5. Engineers mostly use the classical control approach. In control theory, a system is a mechanism, which produce output (endogenous variables) from inputs (exogenous variables). Note finite dimensional in discrete time refer to the state vector that contains a finite number of elements.

From Heij et al. ([15], pp. 6, 7, 16, 82, 151, 158) follows that control systems that are linear $\&$ time invariant (solutions shifted in time remain within the system) can be represented in

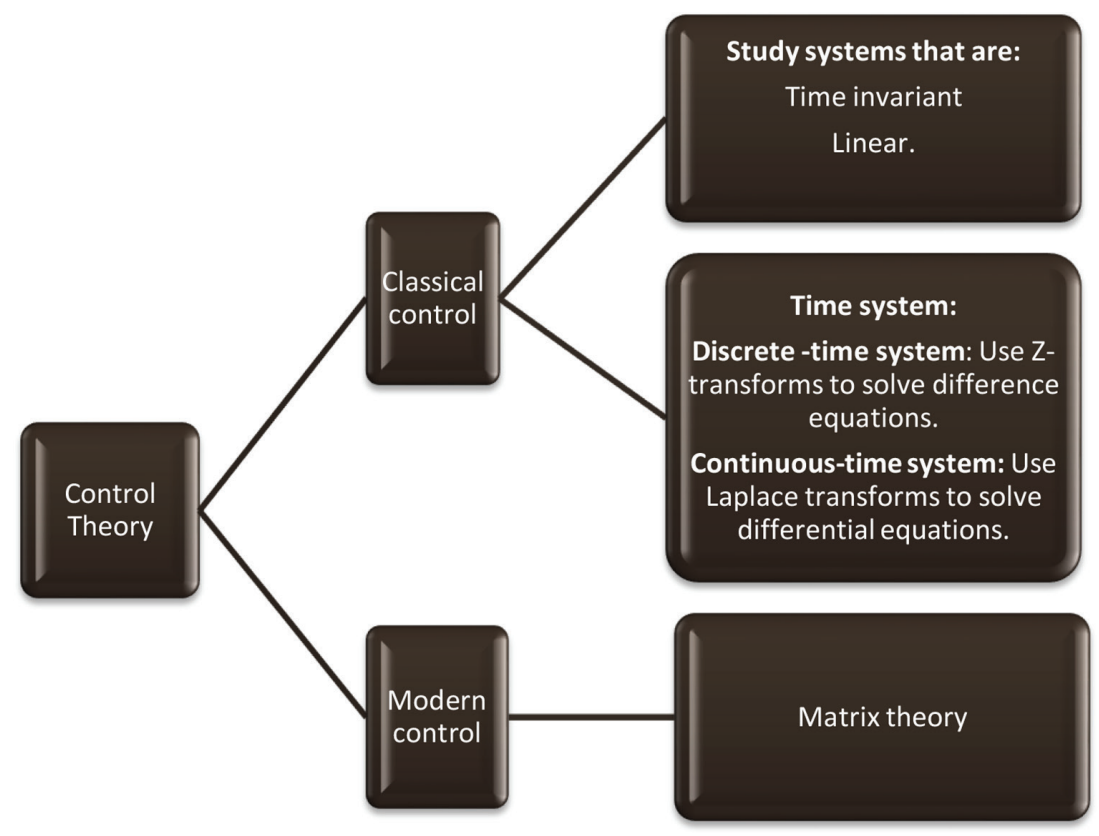

Figure 5: Approach to study control theory.

Source: Own construction from Ref. [15]. 
(finite-dimensional discrete time or causal continuous time or stochastic finite dimensional) state-space form. The discrete time state-space system form is given as

$$
\begin{gathered}
x_{t+1}=\mathrm{A} x_{t}+\mathrm{B} u_{t}, y_{t}=\mathrm{C} x_{t}+\mathrm{D} u_{t}, x_{0}=\text { Value }, t=0,1,2, \ldots ; \\
A=\text { Statetransitionmatrix }, B=\text { Input Matrix }, C=\text { Output matrix }, \\
D=\text { External Matix }, x_{t}=\text { State, } y_{t}=\text { Output }, u_{t}=\text { Input }
\end{gathered}
$$

The (finite-dimensional discrete time) stochastic state-space (input-output) system are of the same form but with output $y_{t}$ an observed stochastic process and input $u_{t}$ an auxiliary white noise process and the state process, $x_{t}$ has the Markov property (i.e. only the present value of a variable is relevant for predicting the future). State-space models can be characterized in terms of transfer (filter) functions. A stochastic system has a finite-dimensional state-space realization if and only if its filter (spectrum) is rational. The class of discrete time stochastic processes that can be represented in state-space form relates to the class of autoregressive moving average (ARMA) processes. From Heij et al. [15], it follow that a strongly stationary discrete time stochastic process, y can be represented in state space form with a stable matrix $\mathrm{A}$ if and only if it can be represented by a strongly stationary ARMA model.

Stability (resilience) is an important objective in state-space systems. Bounded inputs will imply bounded outputs (responses), i.e. a BIBO stable system. Hence, matrix A in the statespace system is stable. Note that there are many other notions of stability, but for finite dimensional (minimal) systems there isn't much difference between many of the stability notions. The unstable state-space system may be stabilised by feedback (state and output), [15]. To fulfil the goal of stability consider:

- If stability is possible by manipulation of the state vector when no control is applied (stability holds in finite-dimensional discrete time if all the eigenvalues of matrix A are in the open unit disc or in the continuous time it means A has all its eigenvalues in the open left half plane, i.e. all roots of the characteristic equation (poles of the transfer/filter function) have negative real parts).

- Is stability possible by manipulation of the inputs (bounded input will lead to bounded output)? The above finite-dimensional discrete time state-space system will be BIBO stable if and only if $\sum_{i=0}^{\infty}\left\|G_{\mathrm{i}}\right\|<\infty$, where $\mathrm{G}_{\mathrm{i}}=C A^{i-1} B$ is the impulse response and $\mathrm{G}_{0}=D$.

For more information on control theory, consider Heij et al. [15] and for infinite dimensional systems, consider Curtain et al. [16].

\subsection{Optimisation}

Optimisation are used in the minimisation or maximisation of some system that might be subject to no constraints or constraints in the form of equality or inequality constraints and/or parameter lower and upper bounds. The optimisation solution depends on the magnitude of the problem in terms of the number of constraints and variables but also on characteristics of the objective function and constraints types. Figure 6 provides more information on the classification of continuous constrained optimisation problems. O'Sullivan 


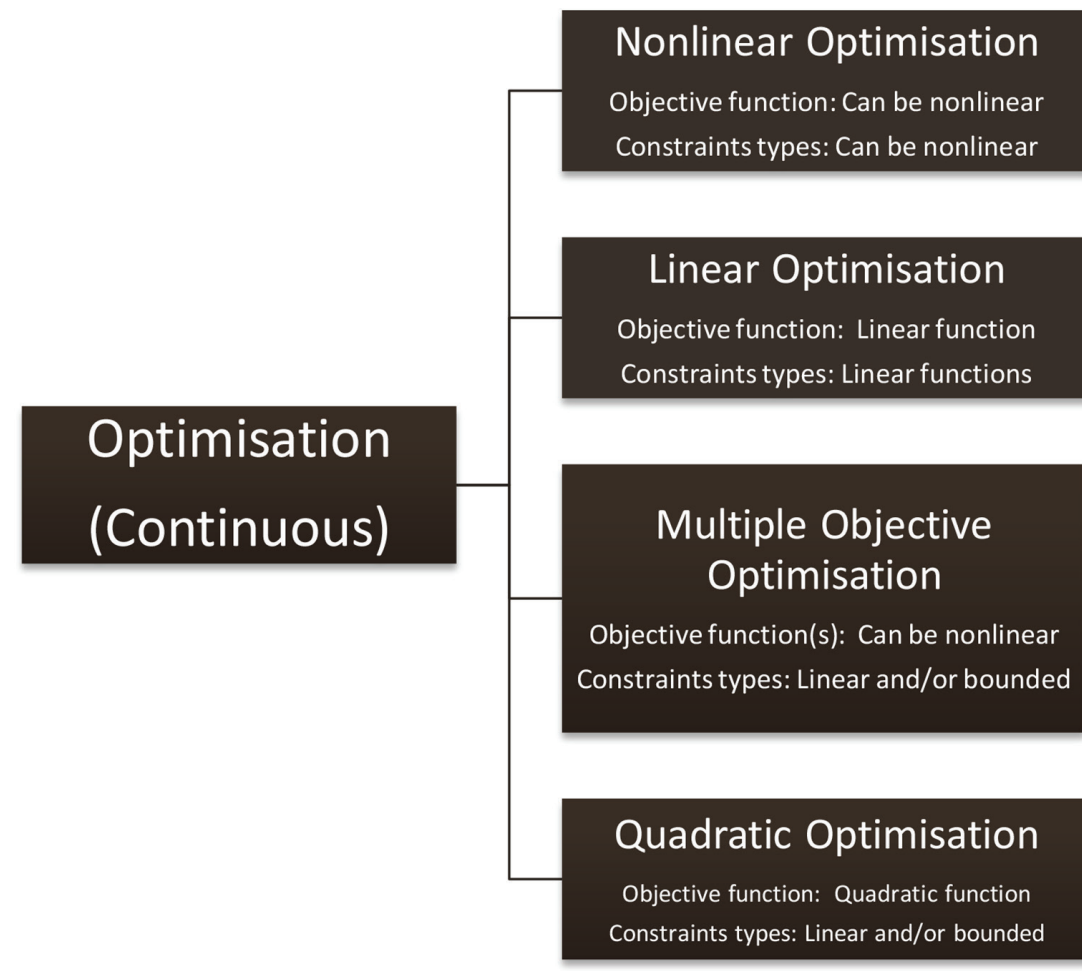

Figure 6: Optimisation taxonomy.

Source: Own construction from Refs. [19] and [20].

[17] used optimisation for road capacity decision. Optimisation can also be used to solve network flow problems, location problems, land use and transport interaction models and in travel demand models. Bottle et al. [18] used a numerical nonlinear optimisation method, i.e. neighbourhood search algorithm to find optimal intervention strategies in the case of a metro system failure.

\section{CONCLUSIONS}

Dynamical systems can be used to describe different and difficult problems in diverse areas but shared characteristics of these systems can be forecasted, modelled and control by the application of the same models or techniques. Different types of models can be used to describe dynamical systems and the model type depends on unintentional particularities and the type of observed data of the problem at hand. Resilience defines how well a system will model uncertainty, defines and forecast the system performance in the presence of disturbances and time delays (output takes time to react).

\section{ACKNOWLEDGEMENTS}

The author gratefully acknowledges the National Research Foundation (GUN No. 81221) for financial support. Any opinion, finding and conclusion or recommendation expressed in this material is that of the author and the NRF does not accept any liability in this regard. 


\section{REFERENCES}

[1] Derissen, S., Quaas, M.F. \& Baumgärtner, S., The relationship between resilience and sustainability of ecological-economic systems. Ecological Economics, 70(6), 11211128, 2011. https://doi.org/10.1016/j.ecolecon.2011.01.003.

[2] Schoeman, C.B., Theoretical perspectives on resilience and sustainability in transportation and spatial planning. WIT SPD Conference, Bristol, 2017.

[3] Schjetlein, T., Haavaldsen, T. \& Lohne, J., Achieving sustainability? A case analysis of policy-to-project processes. Procedia - Social and Behavioral Sciences, 226, pp. 140147, 2016. https://doi.org/10.1016/j.sbspro.2016.06.172

[4] Berdica, K., An introduction to road vulnerability: What has been done, is done and should be done. Transport Policy, 9(2), pp. 117-127, 2002. https://doi.org/10.1016/ s0967-070x(02)00011-2

[5] Anderies, J.M., Folke, C., Walker, B. \& Ostrom, E., Aligning key concepts for global change policy: Robustness, resilience, and sustainability. Ecology and Society, 18(2), p. 8, 2013. http://dx.doi.org/10.5751/ES-05178-180208 [Crossref], [Web of Science ®], [Google Scholar]

[6] Scheinerman, E.R., Invitation to Dynamical Systems. ISBN: 0-13-185000-8.

[7] Hull, J.C., Options, Futures and Other Derivatives, Pearson, 1993. ISBN: 0-13-149908-4.

[8] Rubinstein, R.R., Monte Carlo Optimization, Simulation and Sensitivity of Queueing Networks. Wiley: New York, 1986.

[9] Anderson, G.A., Hoffman, D.L. \& Rasche, R.H., A Vector Error Correction forecasting model of the U.S. economy, pp. 569-598, 2002.

[10] Asteriou, D. \& Hall, S.G., Applied Econometrics, 3rd edn., Published by Palgrave, 2016.

[11] Gujarati, D.N. \& Porter, D.C., Basic Econometrics, 5th edn., Published by McGrawHill Irwin, 2009.

[12] Chu, P.K., Relationship Between Macroeconomic Variables and Net Asset Values (NAV) of Equity Funds: Cointegration Evidence and Vector Error Correction Model of the Hong Kong Mandatory Provident Funds (MPFs), pp. 792-810, 2011.

[13] Oriavwote, V.E. \& Eriemo, N.O., Oil prices and the real exchange rate in Nigeria, pp. 198-205, 2012.

[14] Schoeman I.M., Strategies to reduce traffic accident rates in developing countries: Lessons learned for assessment and management. International Journal of Safety and Security Engineering, 8(1), pp. 98-109, WIT Press, ISSN: 2041-9031, 2018.

[15] Heij, C., Ran, A. \& Van Schagen, F., Introduction to Mathematical Systems Theory: Linear Systems, Identification and Control. ISBN: 13: 978-3-7643-7548-5.

[16] Curtain, R.F. \& Zwart, H.J., An introduction to Infinite-Dimensional Linear Systems Theory, Springer Verlag, 1995.

[17] O'Sullivan, A., Urban Economics, pp. 266-269, McGraw Hill, 2009. ISBN: 978-007-127629-0.

[18] Bottle, M., Di Salvo, C., Placido, A., Motella, B. \& D’ Acierno, L., A neighbourhood search algorithm for determining optimal intervention strategies in the case of metro system failures. International Journal of Transport Development and Integration, 1(1), pp. 63-73, 2017. https://doi.org/10.2495/tdi-v1-n1-63-73

[19] Fletcher, R., Practical Methods of Optimization, Wiley: New York, 1987.

[20] Peressini, A.L., Sullivan, F.E. \& Uhl, J.J., The Mathematics of Nonlinear Programming, Springer-Verlag: New York, 1988. 